Body Image and Body Change Strategies within Friendship Dyads and Groups:

Implications for Adolescent Appearance-Based Rejection Sensitivity

Haley J. Webb

and

Melanie J. Zimmer-Gembeck

School of Applied Psychology and Griffith Health Institute, Behavioral Basis of Health, Griffith University, Australia

\title{
Contact:
}

Haley J Webb

$\mathrm{PhD}$

Griffith University-Gold Coast Campus

School of Psychology

Griffith University QLD 4222

Australia

Tel: 61756788688

e-mail: h.webb@griffith.edu.au 


\begin{abstract}
Appearance-based rejection sensitivity (appearance-RS) consists of concerns about, and expectations of, rejection because of the way one looks (Park, 2007). This study examined dyadic and group level friendship characteristics as correlates of early adolescents' appearance-RS. Using subgroups of an initial sample of 380 participants $\left(M_{\text {age }}=13.84\right)$, appearance-RS was examined within best friend relationships ( $\left.N=132, M_{\text {age }}=13.84\right)$, and friendship groups $\left(N=186, M_{\text {age }}=13.83\right)$. Overall, best friends were found to be similar in their appearance-RS and body dissatisfaction, as well as their restrictive dieting, appearance-conditional self worth, appearance values, and self-rated attractiveness. Similarities between the individual and their friendship group were consistent with the findings for friendship dyads, except for self-rated attractiveness and (unexpectedly) dieting. It was also found that appearance-RS was higher in adolescents whose best friends and friendship groups reported higher levels of restrictive dieting, and self worth that is dependent on feeling attractive. In general, associations did not differ for boys and girls, but having a higher proportion of boys in the friendship group was associated with lowered appearance concerns, perhaps because boys tend to have more positive attitudes toward their appearance.
\end{abstract}




\section{Body Image and Body Change Strategies within Friendship Dyads and Groups: Implications for Adolescent Appearance-Based Rejection Sensitivity}

Attractive individuals are treated more positively, receive more attention, and are perceived as more socially competent, assertive, and intelligent (Langlois et al., 2000; Maner et al., 2003). With first impressions and interpersonal attraction also resting heavily on physical appearance (Feingold, 1990), it seems understandable that many individuals are sensitive to social rejection based on their looks - a processing disposition referred to as appearance-based rejection sensitivity (appearance-RS; Park, 2007). In contrast to the general processing disposition, rejection sensitivity (Downey \& Feldman, 1996), which involves the tendency to anxiously or angrily expect, readily perceive, and overreact to cues of rejection, appearance-RS is focused on when these perceptions are specific to concerns about rejection because of appearance. Individuals with high appearance-RS experience a range of debilitating issues, including depression and social anxiety, poor self-esteem, self-worth that is dependent on feeling attractive, body dissatisfaction, body dysmorphic symptoms, and greater endorsement of cosmetic surgery (Bowker, Thomas, Spencer, \& Park, 2013; Park, 2007, XXX blinded for review).

Although much is known about the adjustment difficulties associated with appearanceRS, only three studies have investigated why appearance-RS varies among adolescents. First, Park, DiRaddo, and Calogero (2009) considered the role of friends in their assessment of sociocultural influences on appearance-RS, and identified that friends' valuing of appearance and perceiving friends' acceptance as being conditional on appearance were linked with appearanceRS in young adults $\left(M d n_{\text {age }}=19\right)$. Second, XXX (blinded for review) showed that appearanceRS was higher in adolescents $\left(M_{\text {age }}=13.8\right)$ who were exposed more often to an "appearance culture” within their friendship context. An appearance culture included appearance 
conversations with friends, pressure to be attractive, distress from appearance teasing, and having friends who valued attractiveness highly. In the third study, Bowker et al. (2013) found that relationships with other-sex peers were associated with appearance-RS and psychological distress in young adolescents $\left(M_{\mathrm{age}}=13.05\right)$, with high acceptance by other-sex peers a risk factor and other-sex friendships a protective factor. Extending upon these findings, the goals of the present study were to more closely examine whether friends are similar in appearance-RS and whether friends’ appearance concerns and behaviours are associated with heightened appearance-RS. This was accomplished by identifying reciprocated dyadic friendships and larger friendship groups, and examining associations within these dyads and groups. Thus, self-reports from each dyad member could be correlated with each other to examine similarity between friends, and selfreports of each friendship group member could be correlated with reports from all other group members.

\section{Best Friends and Friendship Groups}

There are various types of friendship contexts, such as peers, friendship groups, and best friends, and socialisation processes can differ across these types of social systems (Giletta et al., 2012). Within the adolescent friend and peer context, one’s best friend may be a particularly important source of influence. Research shows that individuals make a distinction between the relationship with their best friend, and the relationship with close and casual friends (Fehr, 1996), with close friendships during adolescence often described as more egalitarian and less judgemental than parental relationships (Giordano, 1995). Further, best friend relationships tend to be of higher quality than close friendships (Demir, Ozdemir, \& Weitekamp, 2007). Research shows that friendships that are of higher quality and that are reciprocally engaging - as is expected of best friends - may be the most influential with regards to creating behavioural norms, 
and these friendship norms have been associated with adolescents' greater commitment and adherence to those norms (Dishion \& Tipsord, 2011; Piehler \& Dishion, 2007). However, the connections among larger groups of friends can also be important. It is not uncommon for adolescents to report multiple best or close friends, and many young people do not have a best friend that is stable over even a short length of time (potentially due to the normal instability of early friendships, or the emotional or social difficulties sometimes observed in children without a close friend; Bowker, 2004; Hartup, 1993; 1996). The wider friendship group has been found to play an important role in socialisation processes in many areas, including delinquency, depressive symptoms and religious beliefs (Cheadle \& Schwadel, 2012; Giletta et al., 2012). Thus, our focus on best friends and the wider friendship network permitted identification of the effects of each of these important levels of social influence on adolescents' appearance-RS.

Early adolescence is an important point at which to examine appearance-RS and its social correlates. Early adolescence is characterised by an increase in the saliency of appearance ideals, and physical development that heightens awareness of one's appearance and can contribute to a deviation from appearance ideals (Attie \& Brooks-Gunn, 1989). Thus, young people in this developmental period are at high risk for developing concerns about their appearance and eating problems (Hutchinson \& Rapee, 2007). Moreover, it is during the transition from childhood to adolescence that friends and peers are particularly influential (Thompson et al., 1999). Although people of all ages can be concerned with belonging (Baumeister \& Leary, 1995), significant changes in friendship networks occur during adolescence (La Greca \& Harrison, 2004) with new relationships forming and other relationships ending. By examining appearance-RS within the friendship context among early adolescents, it will be possible to capture the period of development when the transmission of appearance ideals is gaining momentum, concerns about 
acceptance peak, and the combination of these factors may be most critical to the emergence of appearance-RS.

In light of the common concerns about physical appearance, researchers have examined, and verified, the positive association between appearance-RS and body dissatisfaction (Calogero et al., 2010; XXX blinded for review). Researchers propose that adolescents exposed to an appearance-focused friendship context are likely to report heightened body image concerns, due to the sharing and strengthening of attractiveness norms and ideals (Jones, 2004; Webb \& Zimmer-Gembeck, 2013). We speculate that friends’ body image concerns and body change strategies are important contributors to the peer appearance culture, and may show important links with appearance-RS. The relevance of examining body image and body change strategies in our early adolescent sample as contributors to the peer appearance culture is clear when considering that, in multiple Western countries, children as young as six have internalised societal body shape ideals and are aware of dieting as a weight reduction method, and given that concerning levels of body dissatisfaction and various body change strategies are present by adolescence (Lowes \& Tiggemann, 2003; Paxton et al., 1991).

The literature on the role of peers in association with body image and related concerns has tended to focus on individual perceptions of peer influence (Hutchinson \& Rapee, 2007). While it is known that adolescents' subjective views of their friends are important to understanding their own attitudes and behaviours (Zimmer-Gembeck, Hunter, \& Pronk, 2007), examining peer influence on the basis of individual perceptions can be problematic, as these perceptions are subject to inaccuracies, lack of insight, and projection processes (Jaccard, Blanton, \& Dodge, 2005; Zimmer-Gembeck \& Ducat, 2010). Greater insight into the self-reported attitudes and behaviours of friends (and how they may influence one another) could help to better understand the source and the accuracy of young people’s views of their social environment, and inform 
rejection sensitivity and body image interventions that target interactions and behaviours between friends.

\section{Similarity Between Friends and Influence of Friends}

Similarity. Similarity between friends has been theorised to occur via the selection of friends on the basis of similar attitudes or behaviours, and the socialisation toward similarity over time (Ennett \& Bauman, 1994). Whereas similarity in personal-RS was not demonstrated in young adolescent best friends (Bowker, Thomas, Norman, \& Spencer, 2011), researchers have demonstrated similarity between adolescent best friends and within friendship groups across many domains (e.g., Giletta et al., 2012; Van Zalk, Kerr, Branje, Stattin, \& Meeus, 2010). Importantly, adolescent girls have demonstrated similarity to their friends in terms of body dissatisfaction, dietary restraint and extreme weight loss behaviours (Paxton, Schutz, Wertheim, \& Muir, 1999; Woelders, Larsen, Scholte, Cillessen, \& Rutger, 2010). The importance of considering appearance-RS within best friend dyads and friendship groups becomes clear when we consider the socialisation of a wide range of dysfunctional attitudes and behaviours within adolescent peer groups (Dishion \& Tipsord, 2011). Researchers have not explored_the correspondence between adolescents and their friendship groups in their reports of appearanceRS, or the correspondence between best friends in relation to appearance-RS, body dissatisfaction, or eating behaviour. Thus, the first aim of our study was to examine similarity in appearance-RS, as well as related concerns and behaviours, between adolescents and their best friends and friendship groups.

Friend and group correlational effects. Our second aim was to examine associations between adolescent concerns about appearance-based rejection and other appearance-related attitudes and behaviours reported by one's best friend and friendship group. We anticipated that appearance-focused interactions could communicate strong messages to friends about the 
importance of attractiveness, foster the formation of appearance ideals, and encourage selfevaluation through social comparisons. In support of this idea, adolescent body dissatisfaction has been linked with friends' self-reported body dissatisfaction and dietary restraint (Paxton et al., 1999; Woelders et al., 2010). Accordingly, it was expected that appearance-RS would be higher in adolescents whose best friends and friendship groups reported greater body dissatisfaction and restrictive dieting.

It was also expected that 1) the level of appearance-dependent self-worth, 2) physical attractiveness, and 3) the importance placed on appearance reported by adolescents' best friends and friendship group members would be associated with their own self-reported appearance-RS. First, regarding self-worth dependent on attractiveness, Park (2007) highlighted the potentially close association between appearance-RS and appearance-dependent self-worth. Adolescents who place more importance on physical attractiveness for self-worth may encourage their friends to also invest emotionally in their appearance. When feeling attractive is the foundation of one's self-worth, it may be that expectations of social acceptance and approval will also be interpreted in relation to physical appearance. Second, regarding physical attractiveness, researchers have found that individuals feel threatened by friends who are superior to them on a valued trait (Tesser \& Campbell, 1982), and within female friendship pairs it has been found that the less attractive individual perceives more rivalry in the friendship (Bleske-Rechek \& Lighthall, 2010). Thus, a highly attractive friend or friendship group may be experienced as threatening and culminate in greater appearance-RS. Third, perceiving friends to place high importance on appearance has been theorised to encourage adoption of similar attractiveness values, and heighten concerns about being rejected because of an inability to meet society's appearance standards (Park et al., 2009). Empirically, perceptions of friends' appearance-related values have been associated with adolescents' (XXX blinded for review) and young adults' appearance-RS 
(Park et al., 2009). Taken together, these previous findings suggest that early adolescents' appearance-RS may be higher when they have friends who report a higher level of appearancedependent self-worth, when they have friends who report high attractiveness, and when they have friends who place more importance on appearance.

\section{Gender}

Gender differences in the severity of body image and appearance concerns are sometimes reported (Bowker et al., 2013; White \& Halliwell, 2010), as well as differences in how these concerns relate to friend or peer influences (Halliwell \& Harvey, 2006; Jones, Vigfusdottir, \& Lee, 2004). However, XXX (blinded for review) found that associations between adolescent appearance-RS and exposure to friends' appearance-focused interactions did not differ between boys and girls, in general. More broadly, gender differences have been found in the nature of friendship during adolescence and young adulthood. Within young adult same-sex dyads, females engaged more frequently in relationship maintenance behaviours than did males (Oswald, Clark, \& Kelly, 2004), and adolescent girls were found to be more similar to their best friend than were boys, in their psychological distress, behavioural problems, and relationship perceptions (Selfhout, Branje, \& Meeus, 2007). These findings indicate that girls may be more strongly influenced by their best friend and friendship group than boys. Further, as boys tend to show lower rates of appearance-RS, body dissatisfaction and dieting behaviour (Bowker et al., 2013; Wang, Byrne, Kenardy, \& Hills, 2005), a higher percentage of boys within one’s friendship group was expected to be protective of appearance concerns.

\section{Study Aims and Hypotheses}

1. To investigate whether friends would show similarity in appearance-RS. A positive association was expected between best friends' self-reported appearance-RS. It was also 
expected that adolescent appearance-RS would be positively associated with his or her friendship group’s aggregated appearance-RS.

2. To identify whether adolescent appearance-RS would be associated with appearance concerns and behaviours reported by his or her best friend and friendship group. It was expected that appearance-RS would be positively associated with one’s best friend's and friendship group’s body dissatisfaction, restrictive dieting, overexercise, appearanceconditional self-worth, appearance values, attractiveness, and BMI.

3. To investigate whether associations differed according to gender. It was predicted that associations between adolescent appearance-RS and his or her best friend's and friendship group’s body dissatisfaction, restrictive dieting, overexercise, appearance-conditional self-worth, appearance values, attractiveness, and BMI would be stronger in girls than boys. It was also predicted that a higher percentage of boys in one’s friendship group would be associated with lower individual appearance-RS.

\section{Method}

\section{Participants}

Five schools in an urban area of Australia were approached to participate; three schools accepted. Participants were 380 Australian students (43\% male) from one public and two private secondary schools. Across the three schools, participants ranged from low to middle-high income. Participants were aged 12 to 15.5 years $(M=13.84, S D=0.63)$, and were predominantly Caucasian (82.6\%), Asian (7.9\%), or Aboriginal or Pacific Islander (3.4\%). For the dyadic analyses, 132 of the 380 participants were identified as members of reciprocally nominated best friend dyads. Thus, 66 same-sex dyads were formed (27.3\% males), aged 12.7 to 15.5 years $(M=13.8, S D=0.63)$. For friendship group analyses, data from all 380 participants were utilised to form groups and/or in calculating friendship group average scores. However, a 
subset of the total sample was the focus of the group analyses. These were participants who completed a questionnaire, had an identified friendship group with more than one member, and had at least $50 \%$ of their group complete the survey. This resulted in 186 target participants (27.4\% males), aged 12.7 to 15.4 years $(M=13.8, S D=0.60)$. Participants included in the friendship analyses were compared on all demographic measures to excluded participants. Chisquare analysis with follow-up z tests revealed that there was a significantly smaller proportion of individuals who identified as being Aboriginal or Pacific Islander, and a higher proportion of girls than boys included in analyses $(p<.05)$.

To retain the identified subsets of participants in all analyses, multiple imputation was used whenever possible and pooled results are reported. For structural equation modelling, full information maximum likelihood was used to maintain all 132 participants in the APIM's.

\section{Measures}

Best friend nomination. From a provided list of all classmates, participants specified the ID code of their best friend.

Friendship group nominations. A social network assessment measure based on the methods developed by Cairns and colleagues (1985) was used to form friendship groups. Participants were asked to list, using ID codes, groups of children in their grade who hang out together. They were permitted to identify of up to 20 groups each with 20 members, and were prompted to include themselves, dyads, and the same children in different groups if relevant.

Friendship groups were then constructed based on participants' lists of classmates who spent time together (Gest, Farmer, Cairns, \& Xie, 2003). Collecting reports from all participants allowed affiliations between two students to be confirmed across multiple reporters._A computer program (Leung, 1994), based on the Social Cognitive Map procedure (Cairns, Perrin, \& Cairns, 1985), was used to construct groups. This program forms a co-occurrence matrix with each 
participant listed on a row and column. Each matrix cell presents the frequency with which peers nominated each participant as a member of the same group as every other student. Each matrix column provides a co-nomination profile that indicates the frequency with which peers nominated a participant as being in the same group as each of the students in their grade. The diagonal cells present the number of times each participant was nominated as belonging to any group. A correlation matrix is produced by inter-correlating the matrix columns, with the cell contents presenting the similarity between the co-nomination profiles of each participant pair, with higher correlations indicating greater profile similarity (Gest et al., 2003). A reliable affiliation required a correlation $\geq .40$ between two participant profiles (Cairns \& Cairns, 1994). Whereas researchers previously formed discrete groups when exploring the links between body image and related concerns within friendship groups (e.g., Hutchinson \& Rapee, 2007; Paxton et al., 1999), Kindermann (2007) proposed that natural social groups are more complex than this method suggests, as an individual may affiliate with many different individuals and subgroups. Accordingly, unique friendship groups were formed for each target participant by identifying all the individuals with whom they were reliably affiliated. Target participants were included in analyses if at least $50 \%$ of their group completed the survey.

Appearance-RS. Appearance-RS was measured using the Appearance-RS Scale (Park, 2007), which was modified to be age appropriate. For example, "You are at a dance club...” was changed to "You are at a dance/disco...”, and "You are leaving your house to go on a first date...” was changed to "You are leaving your house to go to school...”. Across 10 hypothetical scenarios (e.g., "You are at a dance/disco and all of your friends have been asked to dance except you), participants indicated on a six-point scale their anxiety/concern about appearance-related rejection (e.g., "How concerned or anxious would you be that you have not been chosen to dance because of the way you look?”; 1 = not concerned, 6 = very concerned), and their expectation of 
appearance-related rejection (e.g., "Do you think that it is because of the way you look that no one has asked you to dance?”; 1 = No!!, 6 = Yes!!). Response options two to five on this latter scale were unlabelled, and reflected less definitive responses. To obtain the appearance-RS score, anxious concern was multiplied by expectation of rejection for each item, and these 10 product scores (range 1 to 36) were averaged, with a higher score indicating greater appearanceRS. Cronbach’s $\alpha$ was .89 for girls and .85 for boys.

Body dissatisfaction. The shortened eight-item Body Shape Questionnaire (Evans \& Dolan, 1993) was used to evaluate body dissatisfaction. Participants rated, on a six-point scale (1 = never, 6 = always), how they were feeling about their body weight and shape over the past four weeks (e.g., “Have you felt ashamed of your body?”) Three items were modified to make them suitable for females and males. Items were averaged, and a higher score indicated greater body dissatisfaction (Cronbach's $\alpha=.90$ for girls $\& .88$ for boys).

Disordered body change strategies. To assess disordered eating and exercise habits, the Restraint Scale (Herman \& Polivy, 1975) and the 10-item Excessive Exercise Scale (McCabe \& Vincent, 2009), which were modified for adolescents, were used. The Restraint Scale measured dieting behaviours, concern with food and eating, and history of weight loss and weight gain. Items were averaged to form a total score, and a higher score indicated greater restrictive dieting. Cronbach’s $\alpha$ was .73 for girls and .69 for boys. The Excessive Exercise Scale assessed participants' focus on and need for exercise (e.g., "Do you still exercise if you are tired, injured, or sick?”; 1 = never, 5 = always). Averaging all items formed a total score, and a higher score indicated greater excessive exercise. Cronbach’s $\alpha$ in this sample was .84 for girls and boys.

Appearance-conditional self-worth. The five-item Appearance Subscale of the Contingencies of Self-Worth Scale (Crocker, Luhtanen, Cooper, \& Bouvrette, 2003) was used to 
assess appearance-conditional self-worth (e.g., "When I think I look attractive, I feel good about myself”; 1 = strongly disagree, 5 = strongly agree). Items were averaged to form a total score, and a higher score indicated a stronger appearance-conditional self-worth. Cronbach's $\alpha$ in this sample was .74 for girls and .40 for boys.

Appearance values. A three-item scale was devised to assess participants’ appearance values. Participants rated how much they valued appearance-related characteristics, such as an attractive body or face, and wearing nice clothing ( $1=$ very unimportant, $5=$ very important $)$. Averaging all items created a total score. Cronbach’s $\alpha$ was .89 for girls and .50 for boys.

Attractiveness. An item used by Bleske-Rechek and Lighthall (2010) was employed to assess self-rated attractiveness ("Compared with other girls or boys your age, how physically attractive are you?”; 1 = not at all attractive, 7 = extremely attractive).

BMI. Participants’ height and weight were measured individually, with participant consent, by a trained researcher. These values were used to calculate a body mass index (BMI; weight $\mathrm{kg} /$ height $\mathrm{m}^{2}$ ) for each participant.

\section{Procedure}

Approval for this study was obtained from the University Human Research Ethics Committee. Active parental consent was obtained, with consent forms sent home with children and returned to the school. Students who returned their consent forms were entered into a draw to win a prize, and participating students received a small gift when the survey was completed. Participants completed the questionnaire in their classrooms over two days. The portion of the questionnaire used in the current study took about 45 minutes to complete.

\section{Results}

Table 1 presents the means and standard deviations of participants on all measures. 


\section{Best Friends}

Overview of analyses. Correlations between best friends' scores on each variable were examined to test similarity between best friends. The Actor-Partner Interdependence Model (APIM; e.g., Kashy \& Kenny, 2000) was used to assess the extent to which one person's score on an independent variable was associated with his or her own score on a dependent variable (actor effect), and also his or her best friend's score on the dependent variable (partner effect), while statistically controlling for the non-independence in the two individuals’ responses (Kenny \& Ledermann, 2010). APIMs for indistinguishable dyads were estimated with AMOS software (IBM Corporation). Six equality constraints were applied: equal means and variances of the causal variables, equal intercepts of the outcome variables, equal error variances, equal actor effects, and equal partner effects (Kenny \& Ledermann, 2010). The association of the independent variables with appearance-RS was assessed in seven separate models. Finally, the APIMs predicting appearance-RS were estimated for girls only, to examine whether the associations were stronger than for the whole sample of friendship dyads. As there were only a small number of boy dyads $(n=18)$, gender moderation was not tested. Bootstrapped estimates of paths, standard errors and confidence intervals were reported, but these were similar to the estimates based on maximum likelihood estimation.

\section{Similarity between best friends' appearance-RS and other measures. As predicted}

(Hypothesis 1), best friends' appearance-RS scores were significantly and positively correlated with each others' $(r=.30, p=.01)$. Significant associations were also found between best friends' body dissatisfaction $(r=.29, p<.01)$, restrictive dieting $(r=.36, p<.01)$, overexercise $(r$ $=-.18, p=.04)$, appearance-conditional self-worth $(r=.27, p<.01)$, appearance values $(r=.30, p$ $<.01)$, attractiveness $(r=.28, p<.01)$, and BMI $(r=.35, p<.01)$. 
Actor-Partner Interdependence Models. Table 2 presents the bootstrapped estimates of model paths, standard errors and 95\% confidence intervals of the actor and partner correlational effects. Two of seven possible partner effects were significant (Hypothesis 2). Adolescents' appearance-RS was significantly and positively associated with their best friends' restrictive dieting $(\beta=.16, p<.01)$ and appearance-conditional self-worth $(\beta=.15, p<.01)$. Notably, significant actor effects were found in all APIMs. Appearance-RS was associated with adolescents' own body dissatisfaction $(\beta=.69, p<.01)$, restrictive dieting $(\beta=.52, p<.01)$, overexercise $(\beta=.27, p<.01)$, appearance-conditional self-worth $(\beta=.54, p<.01)$, appearance values $(\beta=.45, p<.01)$, and attractiveness $(\beta=-.29, p<.01)$. All associations were positive except for attractiveness, whereby rating oneself as less attractive was associated with higher appearance-RS. Appearance-RS was not associated with own BMI $(\beta=.02, p=.79)$.

Females only. Because of the smaller set of male relative to female dyads, APIMs were estimated for girls only and compared to the results for the total sample (Hypothesis 3). A very similar pattern of results was produced, and differences between coefficients did not reach significance (all ps >.41). One difference was noted: the negative association between best friends' overexercise in the combined sample $(r=-.18, p<.01)$ was not significant in analyses for girls only $(r=-.08, p=.41)$. Although the difference between -.18 and -.08 was not significant, this finding suggests that the association is not significant in girls and may be more elevated in boys.

\section{Friendship Groups}

Overview of analyses. Unique friendship groups were formed for each target participant by identifying all the individuals with whom each were reliably affiliated. Aggregating the scores of group members, not including the target member, formed friendship group scores for each variable. Characteristics of the friendship groups were examined, and correlations between 
individual scores and the aggregated friendship group scores for each variable were produced. Process (Hayes, 2013) was employed to estimate regression models to test the moderating effect of gender on the associations between individual and friendship group scores.

Friendship group characteristics. The target participants had an average of 12.9 members in their friendship group (ranging from 2 to 32 members). Fifty six percent of groups were homogenous in terms of gender, while the remainder of the groups had a least one girl and one boy.

Similarity between the individual and their friendship group. Table 3 presents correlations between adolescents’ and their friendship groups’ appearance concerns and behaviour. Consistent with expectations (Hypothesis 1), individual appearance-RS was associated with friends’ aggregated, self-reported appearance-RS ( $r=.34, p<.001)$. Associations were also found for body dissatisfaction $(r=.32, p<.001)$, appearance-conditional self-worth $(r=$ $.18, p=.02)$, appearance values $(r=.24, p=.001)$ and BMI $(r=.23, p=.002)$.

\section{Associations of the friendship group's appearance concerns with own appearance-}

RS. As predicted (Hypothesis 2), adolescent appearance-RS was associated with the friendship groups' body dissatisfaction $(r=.28 p<.001)$, restrictive dieting $(r=.24, p<.001)$, appearanceconditional self-worth $(r=.23, p=.001)$, and BMI $(r=-.15, p=.03)$. On the other hand, adolescent appearance-RS was not associated with their friendship groups' overexercise $(r=-.04$, $p=.60)$, appearance values $(r=.04, p=.57)$, and attractiveness $(r=-.10, p=.19)$. The correlations between individual appearance-RS and friends' attitudes and behaviours did not change when controlling for the individual's BMI and the mean of his or her friendship group's BMI ( $p$ s > .42). Further, individual appearance-RS was positively associated with friends’ restrictive dieting $(\beta=.22, p<.001)$ and appearance-conditional self-worth $(\beta=.15, p=.02)$, and negatively associated with the friendship groups’ BMI $(\beta=-.17, p=.03)$, even when controlling 
for the individual's scores on these same measures. Individual appearance-RS was not associated with the friendship groups' body dissatisfaction when controlling for the individual's body dissatisfaction $(\beta=.08, p=.14)$.

Gender. Process (Hayes, 2013) was used to test gender as a moderator of associations of all variables with appearance-RS (Hypothesis 3). No association was moderated by gender (ps>.13). However, as seen in Table 3, individuals with a higher percentage of boys in their friendship group reported lower appearance-RS $(r=-.24, p=.001)$, body dissatisfaction $(r=-.30$, $p<.001)$, restrictive dieting $(r=-.20, p=.007)$, appearance-conditional self-worth $(r=-.28, p$ $<.001)$, and higher levels of overexercise $(r=.15, p=.04)$.

\section{Discussion}

Research into the social correlates of appearance-RS in adolescents and young adults has received only limited research attention (e.g., Bowker et al., 2013; Park et al., 2009). What is emerging from this literature and from body image research, is the key role that friends and peers may play in shaping appearance-related attitudes and behaviours more generally, as well as appearance-RS specifically (Jones, 2004; Jones et al., 2004; Park et al., 2009; XXX, blinded for review). This is the first study to examine both dyadic and group friendship factors that may be linked to the development and perpetuation of appearance-RS in adolescents. Three previous studies have examined portions of this, with one study examining the presence of friendship and peer acceptance as moderators of associations between appearance-RS and psychological functioning in adolescents (e.g., Bowker et al., 2013), and the other two studies examining associations of appearance-RS in adolescents and young adults with perceptions of appearancefocused characteristics of the friend and peer context, including for example appearance conversations with friends, perceived pressure to be attractive, and perceptions that peer acceptance is conditional on appearance (Park et al., 2009; XXX blinded for review). Hence, this 
was the first study that looked at the self-reported appearance concerns and behaviours within best friend dyads and friendship groups, and the implications for adolescent appearance-RS.

\section{Similarity Between Friends}

Researchers have demonstrated friend similarity across many domains, including academic achievement, depression, alcohol consumption, sexual behaviour and self harm (Burgess, Sanderson, \& Umana-Aponte, 2011; Giletta et al., 2012; Van Zalk et al., 2010).

Notably, similarity has been observed within friendship groups in terms of body dissatisfaction and body change strategies (Paxton et al., 1999; Woelders et al., 2010). Consistent with these findings, similarity in appearance-RS between adolescent best friends, and between adolescents and their friendship groups, was found in the present study. Further, best friends demonstrated similarity in their body dissatisfaction, restrictive dieting, appearance-conditional self-worth, appearance values, attractiveness and BMI. Likewise, similarity between adolescents and their friendship groups was demonstrated for body dissatisfaction, appearance-conditional self-worth, appearance values, and BMI, but not restrictive dieting or attractiveness. These results indicate that best friends and friendship groups exhibit similarity in their appearance concerns and values, and body size. These similarities may be due to friendships forming on the basis of pre-existing similarities, through the modelling, reinforcement, and adoption of values and beliefs over time between friends, or due to friends' sharing a socio-cultural environment, fostering comparable experiences and the development of like attitudes (Paxton et al., 1999).

In contrast to previous research that has reported similarity within girls’ friendship groups for dietary restraint and extreme weight loss behaviours (Hutchinson \& Rapee, 2007; Paxton et al., 1999; Woelders et al., 2010), significant similarity was not found in the present study for dieting within friendship groups. This discrepancy may be due to a difference in methodologies. Whereas previous studies formed discrete groups and examined intra- versus inter- friendship 
group variability, we formed unique friendship networks according to each adolescent's' social connections, and correlations were calculated between adolescents' own scores and the average score for the other members of their friendship group. Forming a unique network for each participant permits inclusion of all individuals within a social setting with whom the participant reliability affiliates. In contrast, the process of converting naturally occurring social networks into discrete groups may artificially inflate within-group similarity on some measures. That is, important social connections may be excluded to enable formation of discrete groups, potentially resulting in greater homogeneity. The differential impact of these group formation methodologies requires empirical clarification.

\section{Dyadic and Friendship Group Effects}

Restrictive dieting and overexercise are two behaviours often reported by adolescents as strategies for changing their appearance, and have been associated with serious emotional and physical health concerns, such as binge eating and substance use, emotional stress, and suicide risk (Davis, Blackmore, Katzman, \& Fox, 2005; Lowry, Galuska, Fulton, Burgeson, \& Kann, 2005). Extending upon findings that showed that young people with higher appearance-RS rated themselves as less attractive (Park et al., 2009) and reported greater interest in cosmetic surgery to enhance their appearance (Calogero et al., 2010), the present study found restrictive dieting and overexercise to be elevated in adolescents who reported higher appearance-RS.

Unique to the present study, however, was the investigation of how best friends' and friendship groups' attitudes may be associated with adolescents' appearance-RS, even after considering the roles of personal attitudes about body and appearance. The results showed that appearance-RS was higher in adolescents whose best friends and friendship groups self-reported greater restrictive dieting and greater appearance-dependent self-worth. These associations were strong enough to hold even after controlling for the individual's own attitudes and behaviours. 
Park (2007) drew attention to the parallels between appearance-conditional self-worth and appearance-RS, whereby an individual who bases his or her self-worth on physical appearance may perceive acceptance by others to be similarly determined. Interestingly, sensitivity to appearance-based rejection was positively correlated with the tendency of one's best friend or friendship group to derive self-worth from feelings of attractiveness. In terms of friends' restrictive dieting, findings from the body image literature indicate that adolescents report more body dissatisfaction when their friends or peers engage in dieting or weight loss behaviour (Blowers, Loxton, Grady-Flesser, Occhipinti, \& Dawe, 2003; Lieberman, Gauvin, Bukowski, \& White, 2001), and in a qualitative study, mid-adolescent girls reported that dieting behaviour by close friends made them feel that they, too, ought to be dieting (Wertheim, Paxton, Schutz, \& Muir, 1997). Overall, these findings suggest that restrictive dieting and appearance-conditional self-worth may be communicating powerful messages between adolescent friends about sociocultural standards of beauty and attractiveness, potentially encouraging interpersonal competition, and thus correlating with heightened appearance-RS. In addition, adolescents with heightened appearance-RS may be selecting friends who demonstrate similar concerns and behaviours, including dieting and self-worth that is dependent on appearance.

Interestingly, appearance-RS was higher in adolescents whose friendship groups had lower average BMIs. This result is congruent with the findings of XXX (blinded for review), who found appearance-RS to be higher in girls who rated their friends as more attractive. It may be that a friendship group with a lower BMI is experienced as threatening in comparison to one's own appearance (Bailey \& Ricciardelli, 2010). Thus, having lean friends may elicit greater concerns about being rejected because of one's appearance. Notably, appearance-RS was not associated with best friends' BMI. The higher quality relationship typical of best friends (Demir 
et al., 2007) may protect against the appearance threat posed by having a best friend with a lower BMI and/or having a best friend with a higher BMI may not protect against appearance-RS.

\section{Gender}

Gender differences in the associations between friend or peer influences and body dissatisfaction have been previously reported (Jones et al., 2004; Vincent \& McCabe, 2000), and the literature examining friendship during adolescence and young adulthood suggests that girls may be more strongly influenced by their friends than are boys because their relationships are more intimate and supportive, and they demonstrate better communication in challenging situations (Black, 2000; Oswald et al., 2004; Rose \& Rudolph, 2006). These findings lead one to expect that friends’ appearance-related attitudes and behaviours would be more strongly associated with appearance-RS in girls than in boys, however, dyadic and friendship group results did not support this assumption. Only one gender difference was identified: the negative association between best friends' overexercise found in the combined sample was not significant in the analyses for girls only. The significant, negative association in the combined sample may therefore reflect a negative association in boys, suggesting that the more excessive an adolescent boy's exercise habits become, the less excessive his best friend becomes. However, in light of the small number of boys sampled, this unusual result requires replication.

On the other hand, our results confirmed expectations that a higher percentage of boys within friendship groups would be protective of appearance concerns. This is likely to be due to the lower rates of appearance-RS, body dissatisfaction and dieting found among boys compared to girls (Bowker et al., 2013; Wang et al., 2005). In particular, adolescents with more boys in their friendship group reported lower appearance-RS, as well as lower body dissatisfaction, restrictive dieting, and appearance-conditional self-worth. On the other hand, a higher percentage of boys in the friendship group was associated with greater overexercise. This finding could be 
explained by the higher level of physical activity undertaken in general by boys than girls (te Velde et al., 2007), and is consistent with a previous study that showed adolescent girls’ platonic involvement with boys to be associated with higher body satisfaction (Compian, Gowen, \& Hayward, 2004). Further, the overexercise scale may not be differentiating clearly between high frequency and excessive levels of exercise, as items relating to duration and frequency of exercise, following a regular exercise plan, and the importance of exercise may reflect healthy attitudes toward physical activity.

\section{Limitations and Future Research Directions}

A few study limitations should be noted. First, the small number of male dyads in this study prevented assessment of gender as a moderator of associations between best friends, somewhat weakening our conclusions that associations between best friends and members of friendship groups do not differ according to gender. Our method of having participants name one best friend may have been one reason for the identification of a lower number of boy than girl dyads. Boys’ friendship groups tend to be larger and less intimate (Rose \& Rudolph, 2006), making a single reciprocated nomination less likely. As such, future research might benefit from assessing participants' three closest friends and utilising the first reciprocated match, in order to capture more male dyads in particular. Second, the findings relating to appearance-conditional self-worth should be considered in light of the low internal consistency produced on this measure among boys ( $\alpha=.40)$. This suggests that the items on this measure may not be eliciting a consistent response from boys in this sample, and may require modification when used among early adolescent boys. The most notable study limitation, however, is the cross sectional design, which precludes conclusions about the direction of effects, and mechanisms by which friends’ appearance-related attitudes and behaviours become linked. 
There is one applied implication of the study findings to mention briefly. Having more male friends may be protective of adolescent appearance-RS and related concerns, which could have implications for single-sex schooling. It has been suggested that single-sex girls' schools may pose a risk factor for the development of problematic body image attitudes and eating behaviour through communicating conflicting gender role messages, and due to the greater number of same-sex peers with whom to make appearance comparisons (Carey, Donaghue, \& Broderick, 2013). Our findings suggest that girls’ schools may pose a risk for body image and appearance concerns because their students are prevented from forming potentially protective male friendships within the school environment (Compian et al., 2004). However, as the present study did not specifically evaluate the appearance concerns of students attending single-sex versus co-educational schools, this interpretation requires empirical evaluation.

In summary, the present findings highlight the relevance of relationships with one's best friend and interactions within wider friendship groups during adolescence for understanding sensitivity to appearance-based rejection. Across multiple social system levels, that is at the level of the best friend and friendship group, exposure to friends' appearance concerns, beliefs and body change strategies may create a culture focused on physical appearance within that social system, communicating and reinforcing unhelpful appearance standards, and fostering adolescent concerns about appearance-based social acceptance. 


\section{References}

Attie. I., \& Brooks-Dunn, J. (1989). Development of eating problems in adolescent girls: A longitudinal study. Developmental Psychology, 25, 70-79.

Bailey, S. D., \& Ricciardelli, L. A. (2010). Social comparisons, appearance related comments, contingent self-esteem and their relationships with body dissatisfaction and eating disturbance among women. Eating Behaviors, 11, 107-112.

doi: :1.1016/j.eatbeh.2009.12.001

Baumeister, R. F., \& Leary, M. R. (1995). The need to belong: Desire for interpersonal attachments as a fundamental human motivation. Psychological Bulletin, 3, 497-529.

Black, K. A. (2000). Gender differences in adolescents’ behavior during conflict resolution tasks with best friends. Adolescence, 35, 499-512.

Bleske-Rechek, A., \& Lighthall, M. (2010). Attractiveness and rivalry in women’s friendships with women. Human Nature, 21, 82-97. doi: 1.1007/s12110-010-9081-5

Blowers, L. C., Loxton, N. J., Grady-Flesser, M., Occhipinti, S., \& Dawe, S. (2003). The relationship between sociocultural pressure to be thin and body dissatisfaction in preadolescent girls. Eating Behaviors, 4, 229-244. doi: 1.1016/S1471-0153(03)00018-7

Bowker, A. (2004). Predicting friendship stability during early adolescence. The Journal of Early Adolescence, 24, 85-112. doi: 10.1177/0272431603262666

Bowker, J. C., Thomas, K. K., Norman, K. E., \& Spencer, S. V. (2011). Mutual best friendship involvement, best friend rejection sensitivity, and psychological maladaptation. Journal of Youth and Adolescence, 40, 545-555. doi: 1.1007/s10964-010-9582-x

Bowker, J. C., Thomas, K. K., Spencer, S. V., \& Park. L. E. (2013). Examining appearance-based rejection sensitivity during early adolescence. Journal of Research on Adolescence, 23, 375-388. doi:†1.1111/jora.12003 
Burgess, S., Sanderson, E., \& Umana-Aponte, M. (2011). School ties: An analysis of homophily in an adolescent friendship network. University of Bristol. Retrieved from http://www.bristol.ac.uk/cmpo/publications/papers/2011/wp267.pdf

Cairns, R. B., \& Cairns, B. D. (1994). Lifelines and risks: Pathways of youth in our time. Cambridge: Cambridge University Press.

Cairns, R. B., Perrin, J. E., \& Cairns, B. D. (1985). Social structure and social cognition in early adolescence: Affiliative patterns. Journal of Adolescence, 5, 339-355. doi: $1.1177 / 0272431685053007$

Calogero, R. M., Park, L. E., Rahemtulla, Z. K., \& Williams, K. C. D. (2010). Predicting excessive body image concerns among British university students: The unique role of appearance-based rejection sensitivity. Body Image, 7, 78-81. doi: 1.1016/j.bodyim.2009.09.005

Carey, R. N., Donaghue, N., \& Broderick, P. (2011). 'What you look like is such a big factor': Girls' own reflections about the appearance culture in an all-girls' school. Feminism \& Psychology, 21, 299-316. doi: 10.1177/0959353510369893

Cheadle, J. C., \& Schwadel, P. (2012). The 'friendship dynamics of religion,' or the 'religious dynamics of friendship’? A social network analysis of adolescents who attend small schools. Social Science Research, 41, 1198-1212. doi: 1.1016/j.ssresearch.2012.03.014

Compian, L., Gowen, L. K., \& Hayward, C. (2004). Peripubertal girls’ romantic and platonic involvement with boys: Associations with body image and depression symptoms. Journal of Research on Adolescence, 14, 23-470. doi: 10.1111/j.1532-7795.2004.01401002.x

Crocker, J., Luhtanen, R. K., Cooper, M. L., \& Bouvrette, A. (2003). Contingencies of self-worth in college students: Theory and measurement. Journal of Personality and Social Psychology, 85, 894-908. doi: 1.1037/0022-3514.85.5.894 
Davis, C., Blackmore, E., Katzman, D. K., \& Fox, J. (2005). Female adolescents with anorexia nervosa and their parents: A casecontrol study of exercise attitudes and behaviours. Psychological Medicine, 35, 377-386. doi: 1.1017/S0033291704003447

Demir, M., Ozdemir, M., \& Weitekamp, L. A. (2007). Looking to happy tomorrows with friends: Best and close friendships as they predict happiness. Journal of Happiness Studies, 8, 243-271. doi: 1.1007/s10902-006-9025-2

Dishion, T. J., \& Tipsord, J. M. (2011). Peer contagion in child and adolescent social and emotional development. Annual Review of Psychology, 62, 189-214.

doi: 1.1146/annurev.psych.093008.100412

Downey, G., \& Feldman, S. I. (1996). Implications of rejection sensitivity for intimate relationships. Journal of Personality and Social Psychology, 70, 1327-1343. doi: 1.1037/0022-3514.7.6.1327

Ennett, S. T., \& Bauman, K. E. (1994). The contribution of influence and selection to adolescent peer group homogeneity: The case of adolescent cigarette smoking. Journal of Personality and Social Psychology, 67, 653-663. doi: 1.1037/0022-3514.67.4.653

Evans, C., \& Dolan, B. (1993). Body Shape Questionnaire: Derivation of shortened "alternate forms". International Journal of Eating Disorders, 13, 315-321. doi: 1.1002/1098108X(199304)13:3<315::AID-EAT2260130310>3..CO;2-3

Fehr, B. A. (1996). Friendship processes. Thousand Oaks, Calif: Sage Publications

Feingold, A. (1990). Gender differences in effects of physical attractiveness on romantic attraction: A comparison across five research paradigms. Journal of Personality and Social Psychology, 59, 981-993. 
Gest, S. D., Farmer, T. W., Cairns, B. D. \& Xie, H. (2003). Identifying children’s peer social networks in school classrooms: Links between peer reports and observed interactions. Social Development, 12, 513-529. doi: 1.1111/1467-9507.00246

Giletta, M., Scholte, R. H. J., Prinstein, M. J., Engels, R. C. M. E., Rabaglietti, E., \& Burk, W. J. (2012). Friendship context matters: Examining the domain specificity of alcohol and depression socialization among adolescents. Journal of Abnormal Child Psychology, 40, 1027-1043. doi: 1.1007/s10802-012-9625-8

Giordano, P. C. (1995). The wider circle of friends in adolescence. The American Journal of Sociology, 101, 661-697. Retrieved from: http://www.jstor.org/stable/2781997

Halliwell, E., \& Harvey, M. (2006). Examination of a sociocultural model of disordered eating among male and female adolescents. British Journal of Health Psychology, 11, 235-248. doi: 1.1348/135910705X39214

Hartup, W. W. (1993). Adolescents and their friends. New Directions for Child and Adolescent Development, 60, 3-22. doi: 1.1002/cd.23219936003

Hartup, W. W. (1996). The company they keep: Friendships and their developmental significance. Child Development, 67, 1-13. Retrieved from http://www.jstor.org/stable/1131681.

Hayes, A. F. (2013). Introduction to mediation, moderation, and conditional process analysis: A regression-based approach. New York: Guilford Press.

Herman, C. P., \& Polivy, J. (1975). Anxiety, restraint, and eating behavior. Journal of Abnormal Psychology, 84, 666-672.

Hutchinson, D. M., \& Rapee, R. M. (2007). Do friends share similar body image and eating problems? The role of social networks and peer influences in early adolescence. Behavior Research and Therapy, 45, 1557-1577. doi: 1.1016/j.brat.2006.11.007 
Jaccard, J., Blanton, H., \& Dodge, T. (2005). Peer influences on risk behavior: An analysis of the effects of a close friend. Developmental Psychology, 41, 135 - 147. doi: 1.1037/00121649.41.1.135

Jones, D. C. (2004). Body image among adolescent girls and boys: A longitudinal study. Developmental Psychology, 40, 823-835. doi: 1.1037/0012-1649.4.5.823

Jones, D. C., Vigfusdottir, T. H., \& Lee, Y. (2004). Body image and the appearance culture among adolescent girls and boys: An examination of friend conversations, peer criticism, appearance magazines, and the internalization of appearance ideals. Journal of Adolescent Research, 19, 323-339. doi: 1.1177/0743558403258847

Kashy, D. A., \& Kenny, D. A. (2000). The analysis of data from dyads and groups. In H. Reis \& C. M. Judd (Eds.), Handbook of research methods in social and personality psychology (pp. 451-477). New York: Cambridge University Press.

Kenny, D. A., \& Ledermann, T. (2010). Detecting, measuring, and testing dyadic patterns in the Actor-Partner Interdependence Model. Journal of Family Psychology, 24, 359-366. doi: $1.1037 / \mathrm{a} 0019651$

Kindermann, T. A. (2007). Effects of naturally existing peer groups on changes in academic engagement in a cohort of sixth graders. Child Development, 78, 1186-1203. doi: 1.1111/j.1467-8624.2007.0106.x

La Greca, A. M., \& Harrison, H. M. (2005). Adolescent peer relations, friendships, and romantic relationships: Do they predict social anxiety and depression? Journal of Clinical Child and Adolescent Psychology, 34, 49-61. doi: 10.1207/s15374424jccp3401_5

Langlois. J. H., Kalakanis, L., Rubenstein, A. J., Larson, A., Hallam, M., \& Smoot, M. (2000). Maxims or myths of beauty? A meta-analytic and theoretical review. Psychological Bulletin, 126, 390-423. doi: 1.1037/0033-2909.126.3.390 
Leung, M. (1994). Social cognitive map, version 3.2. Chapel Hill: Department of Psychology, University of North Carolina

Lieberman, M., Gauvin, L., Bukowski, W. M., \& White, D. R. (2001). Interpersonal influence and disordered eating behaviors in adolescent girls: The role of peer modeling, social reinforcement, and body-related teasing. Eating Behaviors, 2, 215-236. doi: $1.1016 /$ S1471-0153(01)00030-7

Lowes, J., \& Tiggemann, M. (2003). Body dissatisfaction, dieting awareness and the impact of parental influence in young children. British Journal of Health Psychology, 8, 135-147. doi: 10.1348/135910703321649123

Lowry, R., Galuska, D. A., Fulton, J. E., Burgeson, C. R., \& Kann, L. (2005). Weight management goals and use of exercise for weight control among U.S. high school students, 1991-2001. Journal of Adolescent Health, 36, 320-326. doi: 1.1016/j.jadohealth.2004.03.010

Maner, J. K., Kenrick, D. T., Becker, D. V., Delton, A. W., Hofer, B., Wilbur, C. J., \& Neuberg, S. L. (2003). Sexually selective cognition: Beauty captures the mind of the beholder. Journal of Personality and Social Psychology, 85, 1107-112. doi: 1.1037/00223514.85.6.1107

McCabe, M. P., \& Vincent, M. A. (2009). Development of body modification and excessive exercise scales for adolescents. Assessment, 9, 131-141. doi: 1.1177/10791102009002003

Oswald, D. L., Clark, E. M., \& Kelly, C. M. (2004). Friendship maintenance: An analysis of individual and dyad behaviors. Journal of Social and Clinical Psychology, 23, 413-441. doi: $1.1521 /$ jscp.23.3.413.35460

Park, L. E. (2007). Appearance-based rejection sensitivity: Implications for mental and physical health, affect, and motivation. Personality and Social Psychology Bulletin, 33, 490-504. 
doi: $1.1177 / 0146167206296301$

Park, L. E., DiRaddo, A. M., \& Calogero, R. M. (2009). Sociocultural influence and appearancebased rejection sensitivity among college students. Psychology of Women Quarterly, 33, 108-119. doi: 1.1111/j.1471-6402.2008.01478.x

Paxton, S. J., Schutz, H. K., Wertheim, E. H., \& Muir, S. L. (1999). Friendship clique and peer influences on body image concerns, dietary restraint, extreme weight-loss behaviors, and binge eating in adolescent girls. Journal of Abnormal Psychology, 108, 255-266. doi: 1.1037/0021-843X.108.2.255

Paxton S. J., Wertheim, E. H., Gibbons, K., Szmukler, G. I., Hillier, L., \& Petrovich, J. L. (1991). Body image satisfaction, dieting beliefs, and weight loss behaviors in adolescent girls and boys. Journal of Youth and Adolescence, 20, 361-379. doi: 10.1007/BF01537402

Piehler, T. F., \& Dishion, T. J. (2007). Interpersonal dynamics within adolescent friendship: Dyadic mutuality, deviant talk, and patterns of antisocial behavior. Child Development, 78, 1611-1624. doi: 10.1111/j.1467-8624.2007.01086.x

Prinstein, M. J., \& Wang, S. S. (2005). False consensus and adolescent peer contagion: Examining discrepancies between perceptions and actual reported levels of friends‘ deviant and health risk behaviors. Journal of Abnormal Child Psychology, 33, 293-306. doi: 10.1007/s10802-005-3566-4

Rose, A. J., \& Rudolph, K. D. (2006). A review of sex differences in peer relationship processes: Potential trade-offs for the emotional and behavioral development of girls and boys. Psychological Bulletin, 132, 98-131. doi: 1.1037/0033-2909.132.1.98

Selfhout, M. H. W., Branje, S. J. T., \& Meeus, W. H. J. (2007). Similarity in adolescent best friends: The role of gender. Netherlands Journal of Psychology, 63, 42-48. doi: 1.1007/BF03061061 
Swami, V., \& Tovee, M. J. (2005a). Female physical attractiveness in Britain and Malaysia: A cross-cultural study. Body Image, 2, 115-128. doi: 1.1016/j.bodyim.2005.02.002

Swami, V., \& Tovee, M. J. (2005b). Male physical attractiveness in Britain and Malaysia: A cross-cultural study. Body Image, 2, 383-393. doi: 1.1016/j.bodyim.2005.08.001

te Velde, S. J., De Bourdeaudhuij, I., Thorsdottir, I., Rasmussen, M., Hagstromer, M., Klepp, K., \& Brug, J. (2007). Patterns in sedentary and exercise behaviors and associations with overweight in 9-14-year-old boys and girls - a cross-sectional study. BMC Public Health, 7(16). doi: 1.1186/1471-2458-7-16

Tesser, A., \& Campbell, J. (1982). Self-evaluation maintenance and the perception of friends and strangers. Journal of Personality, 50, 261-279. doi: 1.1111/j.1467-6494.1982.tb0075.x

Thompson, J. K., Heinberg, L. J., Altabe, M., \& Tantleff-Dunn, S. (1999). Exacting beauty: Theory, assessment, and treatment of body image disturbance. Washington, DC: American Psychological Association.

Van Zalk, M. H. W., Kerr, M., Branje, S. J. T., Stattin, H., \& Meeus, W. H. J. (2010). It takes three: Selection, influence, and de-selection processes of depression in adolescent friendship networks. Developmental Psychology, 46, 927-938. doi: 1.1037/a0019661

Vincent, M. A., \& McCabe, M. P. (2000). Gender differences among adolescents in family, and peer influences on body dissatisfaction, weight loss, and binge eating behaviors. Journal of Youth and Adolescence, 29, 205-221. doi: 1.1023/A:1005156616173

Wang, Z., Byrne, N. M., Kenardy, J. A., \& Hills, A. P. (2005). Influences of ethnicity and socioeconomic status on the body dissatisfaction and eating behaviour of Australian children and adolescents. Eating Behaviors, 6, 23-33. doi: 1.1016/j.eatbeh.2004.05.001 
Wertheim, E. H., Paxton, S. J., Schutz, H. K., \& Muir, S. L. (1997). Why do adolescent girls watch their weight? An interview study examining sociocultural pressures to be thin. Journal of Psychosomatic Research, 42, 345-355. doi: 1.1016/S0022-3999(96)00368-6

White, J., \& Halliwell, E. (2010). Examination of a sociocultural model of excessive exercise among male and female adolescents. Body Image, 7, 227-233.

doi: 1.1016/j.bodyim.201.02.002

Woelders, L. C. S., Larsen, J. K., Sholte, R. H. J., Cillessen, A. H. N., \& Engels, R. C. M. E. (2010). Friendship group influences on body dissatisfaction and dieting among adolescent girls: A prospective study. Journal of Adolescent Health, 47, 456-462. doi: 1.1016/j.jadohealth.201.03.006

Zimmer-Gembeck, M. J., \& Ducat, W. (2010). Positive and negative romantic relationship quality: Age, familiarity, attachment and well-being as correlates of couple agreement and projection. Journal of Adolescence, 33, 879-890.

Zimmer-Gembeck, M. J., Hunter, T. A., \& Pronk, R. (2007). A model of behaviors, peer relations and depression: Perceived social acceptance as a mediator and the divergence of perceptions. Journal of Social and Clinical Psychology, 26, 273-302.

doi: $1.1521 /$ jscp.2007.26.3 
Table 1

Means and Standard Deviations on all Measures

\begin{tabular}{lcc}
\hline & $\begin{array}{c}\text { Friendship group sample } \\
M(S D)\end{array}$ & $\begin{array}{c}\text { Dyadic sample } \\
(S D)\end{array}$ \\
\hline Age & $13.83(0.60)$ & $13.85(0.63)$ \\
Appearance-RS & $9.38(6.08)$ & $10.71(6.16)$ \\
Body dissatisfaction & $2.11(0.99)$ & $2.34(1.09)$ \\
Restrictive Dieting & $1.90(0.40)$ & $1.94(0.43)$ \\
Overexercise & $2.92(0.74)$ & $2.89(0.69)$ \\
Appearance-conditional self-worth & $3.11(0.73)$ & $3.29(0.71)$ \\
Appearance values & $3.07(0.90)$ & $3.25(0.89)$ \\
Attractiveness & $4.00(1.09)$ & $3.92(1.17)$ \\
BMI & $20.99(3.07)$ & $20.53(3.12)$ \\
\hline
\end{tabular}


Table 2

Bootstrapped Estimates from The Actor-Partner Interdependence Models of Appearance-RS $(N=66$ dyads $)$

\begin{tabular}{|c|c|c|c|c|c|}
\hline \multirow[b]{2}{*}{ Variables } & \multicolumn{2}{|c|}{ Unstandardised } & \multicolumn{3}{|c|}{ Standardised } \\
\hline & Estimate & $\mathrm{SE}$ & Estimate & SE & $p$ \\
\hline \multicolumn{6}{|l|}{ Actor Effect } \\
\hline \multicolumn{6}{|l|}{ Own: } \\
\hline Body dissatisfaction & 3.89 & .25 & .69 & .03 & $<.01$ \\
\hline Restrictive dieting & 7.43 & .70 & .52 & .04 & $<.01$ \\
\hline Overexercise & 2.35 & .51 & .27 & .06 & $<.01$ \\
\hline Appearance-conditional self-worth & 4.70 & .48 & .54 & .05 & $<.01$ \\
\hline Appearance values & 3.12 & .41 & .45 & .05 & $<.01$ \\
\hline Attractiveness & -1.53 & .33 & -.29 & .06 & $<.01$ \\
\hline BMI & 0.03 & .11 & .02 & .06 & .79 \\
\hline \multicolumn{6}{|l|}{ Partner Effect } \\
\hline \multicolumn{6}{|l|}{ Best friend's: } \\
\hline Body dissatisfaction & 0.40 & .30 & .07 & .05 & .16 \\
\hline Restrictive dieting & 2.22 & .75 & .16 & .05 & $<.01$ \\
\hline Overexercise & -0.33 & .51 & -.04 & .06 & .53 \\
\hline Appearance-conditional self-worth & 1.31 & .41 & .15 & .05 & $<.01$ \\
\hline Appearance values & 0.61 & .35 & .09 & .05 & .07 \\
\hline Attractiveness & 0.26 & .28 & .05 & .05 & .43 \\
\hline $\mathrm{BMI}$ & -0.07 & .10 & -.04 & .05 & .49 \\
\hline
\end{tabular}


Table 3

Correlations Between Individual and Friendship Group Scores $(N=186)$

\begin{tabular}{|c|c|c|c|c|c|c|c|c|c|}
\hline \multirow[b]{2}{*}{ Individual appearance concerns: } & \multicolumn{8}{|c|}{ Friends' appearance concerns } & \multirow{2}{*}{$\begin{array}{c}\text { \% male in } \\
\text { group }\end{array}$} \\
\hline & 1 & 2 & 3 & 4 & 5 & 6 & 7 & 8 & \\
\hline 1. Appearance-RS & $.34 * * *$ & $.29 * * *$ & $.26 * * *$ & .04 & $.23 * *$ & .04 & -.10 & $-.16^{*}$ & $-.24 * *$ \\
\hline 2. Body dissatisfaction & & $.32 * * *$ & .14 & .04 & $.28 * * *$ & .06 & -.10 & -.13 & $-.30 * * *$ \\
\hline 3. Restrictive dieting & & & .06 & .04 & .12 & .07 & $-.16 *$ & -.07 & $-.20 * *$ \\
\hline 4. Overexercise & & & & .12 & -.06 & -.03 & $.17^{*}$ & -.12 & $.15^{*}$ \\
\hline 5. Appearance-conditional self-worth & & & & & $.18^{*}$ & -.02 & -.10 & -.10 & $-.28 * * *$ \\
\hline 6. Appearance values & & & & & & $.24 * *$ & .03 & -.04 & .09 \\
\hline 7. Attractiveness & & & & & & & .12 & -.02 & .11 \\
\hline 8. BMI & & & & & & & & $.23 * *$ & .13 \\
\hline
\end{tabular}

${ }^{*} p<.05 .{ }^{* *} p<.01 .{ }^{* * *} p<.001$. 